\title{
Participation of Transmembrane Domain 1 of Presenilin 1 in the Catalytic Pore Structure of the $\gamma$-Secretase
}

\author{
Shizuka Takagi (高木穏香), ${ }^{1}$ Aya Tominaga (富永綾), ${ }^{1}$ Chihiro Sato (佐藤千尋), ${ }^{1,2,3}$ Taisuke Tomita (富田泰輔), ${ }^{1,2}$ \\ and Takeshi Iwatsubo (岩坪威) ${ }^{1,2,3}$ \\ ${ }^{1}$ Department of Neuropathology and Neuroscience, Graduate School of Pharmaceutical Sciences, The University of Tokyo, Tokyo 113-0033, Japan, ${ }^{2}$ Core \\ Research for Evolutional Science and Technology, Japan Science and Technology Corporation, and ${ }^{3}$ Department of Neuropathology, Graduate School of \\ Medicine, The University of Tokyo, Hongo 7-3-1, Bunkyo, Tokyo 113-0033, Japan
}

$\gamma$-Secretase is an intramembrane-cleaving protease that is responsible for the generation of amyloid- $\beta$ peptides linked to the pathogenesis of Alzheimer's disease. Using a substituted cysteine accessibility method, we have previously shown that the hydrophilic "catalytic pore" structure of $\gamma$-secretase is formed by the transmembrane domains (TMDs) 6, 7, and 9 of presenilin 1 (PS1), the catalytic subunit of $\gamma$-secretase, within the membrane. Here, we analyzed the structure in and around the first hydrophobic region, the putative TMD1, of PS1, of which the precise function as well as three-dimensional location within $\gamma$-secretase remained unknown. We found that TMD1 is located in proximity to the catalytic GxGD and PAL motifs within the C-terminal fragment of PS1, facing directly the catalytic pore. Competition experiments using known $\gamma$-secretase inhibitors suggested that the $\mathrm{N}$-terminal region of TMD1 functions as a subsite during proteolytic action of the $\gamma$-secretase. Intriguingly, binding of inhibitors affected water accessibility of residues at the membrane border of TMD1, suggesting the possibility of a dynamic motion of TMD1 during the catalytic process. Our results provide mechanistic insights into the functional role of TMD1 of PS1 in the intramembrane-cleaving activity of the $\gamma$-secretase.

\section{Introduction}

The process of generation and deposition of amyloid- $\beta$ peptide $(\mathrm{A} \beta)$ is strongly implicated in the pathogenesis of Alzheimer/s disease (AD) (Haass and Selkoe, 2007). Accordingly, $\gamma$-secretase, a protease responsible for the $\mathrm{A} \beta$ generation, is one of the plausible therapeutic targets for AD (Tomita, 2009; De Strooper et al., 2010). However, $\gamma$-secretase cleaves several type I single-span membrane proteins, including Notch receptor. Thus, the mechanism-based rational design of $\gamma$-secretase inhibitors (GSIs) based on the structural information of the $\gamma$-secretase is unequivocally needed for development of $\mathrm{AD}$ therapeutics. $\gamma$-Secretase is an unconventional aspartyl protease that hydrolyzes its substrates within the hydrophobic lipid bilayer. It belongs to a unique group of intramembrane-cleaving proteases (I-CLiPs), of which site 2 protease (S2P), signal peptide peptidase, and rhomboid have been identified as other members

\footnotetext{
Received June 26, 2010; revised Sept. 12, 2010; accepted Sept. 23, 2010.

This work was supported by Grants-in-Aid for Young Scientists (S) (to T.T.) from the Ministry of Education, Culture, Sports, Science, and Technology of Japan (MEXT), by Scientific Research on Priority Areas "Research on Pathomechanisms of Brain Disorders" from MEXT (to T.T., T.I.), by the Program for Promotion of Fundamental Studies in Health Sciences of the National Institute of Biomedical Innovation (to T.T., T.I.), by Targeted Proteins Research Program of the Japan Science and Technology Corporation (JST) (to T.T., T.I.), and by Core Research for Evolutional Science and Technology of JST (to T.T., T.I.), Japan. S.T. and C.S. are research fellows of the Japan Society for the Promotion of Science. We thank Drs. G. Thinakaran (University of Chicago, Chicago, IL) for antibodies, B. De Strooper (KU Leuven, Leuven, Belgium) for DKO cells, T. Kitamura (University of Tokyo, Tokyo, Japan) for retroviral infection system, and Takeda Pharmaceutical Company for A $\beta$ ELISA. We are also grateful to our laboratory members for helpful discussions and technical assistance.

Correspondence should be addressed to Dr. Taisuke Tomita, Department of Neuropathology and Neuroscience, Graduate School of Pharmaceutical Sciences, The University of Tokyo, 7-3-1 Hongo, Bunkyo-ku, Tokyo 113-0033, Japan. E-mail: taisuke@mol.f.u-tokyo.ac.jp.

DOI:10.1523/JNEUROSCI.3318-10.2010

Copyright $\odot 2010$ the authors $\quad 0270-6474 / 10 / 3015943-08 \$ 15.00 / 0$
}

(Wolfe and Kopan, 2004). The recently resolved crystal structures of the rhomboid and S2P have shed light on the possible mechanism as to how peptide bond hydrolysis can take place within the lipid environment (Urban and Shi, 2008). Nonetheless, structural analysis such as x-ray crystallography is still an extremely challenging task for multimeric membrane protein complexes (e.g., the $\gamma$-secretase), which is comprised of nicastrin, anterior pharynx defective-1, presenilin enhancer-2, and PS (Takasugi et al., 2003). We have been applying substituted cysteine accessibility method (SCAM) to gain insights into the structure of presenilin 1 (PS1) in a membrane-embedded state. SCAM has been reiteratively used to obtain structural information of various multipass membrane proteins in a functional state, by covalently modifying the introduced cysteine (Cys) residues using sulfhydryl reagents (Karlin and Akabas, 1998; Kaback et al., 2001). Using SCAM, we and others have revealed that PS1 harbors a hydrophilic "catalytic pore" formed by transmembrane domains (TMDs) 6, 7, and 9 (Sato et al., 2006, 2008; Tolia et al., 2006, 2008). Recent nuclear magnetic resonance analysis of the C-terminal region of PS1 supported the results of SCAM analysis (Sobhanifar et al., 2010). This was consistent with the structures obtained from the crystallographic analyses of rhomboid and $\mathrm{S} 2 \mathrm{P}$, further suggesting that the hydrophilic milieu around the active site located within the lipid bilayer is a common structure across I-CLiPs. Meanwhile, recent chemical biological studies revealed that several GSIs directly target the N-terminal fragment (NTF) of PS1 (Fuwa et al., 2007; Imamura et al., 2009). Thus, an important remaining question regarding the structure-function relationship of $\gamma$-secretase is how the other five TMDs within the PS1 NTF contribute to the $\gamma$-secretase activity. Here, we used 
SCAM and other biochemical methods to analyze the structure and the functional role of TMD1 of PS1, which has been shown to be indispensable for the stability, as well as the catalytic activity, of the $\gamma$-secretase (Watanabe et al., 2005, 2010).

\section{Materials and Methods}

G1L3, which recognizes the hydrophilic loop 6 of PS1, was described previously (Tomita et al., 1999). Anti-PS1 $1_{\mathrm{NT}}$ (Thinakaran et al., 1998) was kindly provided by Dr. Thinakaran (University of Chicago, Chicago, IL). Other antibodies were purchased from BD Biosciences (anti-E-cadherin), Cell Signaling Technology [anti-cleaved Notch1 (V1744) and anti-myc tag (9B11)], or Sigma [anti$\alpha$-tubulin (DM1A)]. \{1S-Benzyl-4R-[1-(1S-carbamoyl-2 - phenylethylcarbamoyl) - $1 S$-3methyl-butylcarbamoyl]-2 R-hydroxy-5phenylpentyl $\}$ carbamic acid tert-butyl ester (L-685,458) (Shearman et al., 2000) and peptide 15 (pep15) (Das et al., 2003) were purchased from Bachem and BEX, respectively. $N-[N-(3,5-$ difluorophenacetyl)]-L-alanyl]-S-phenylglycine tert-butyl ester (DAPT) was synthesized as described previously (Dovey et al., 2001; Kan et al., 2004). All 3-(4,5-dimethylthiazol-2-yl)-5-(3carboxymethoxyphenyl)-2-(4-sulfophenyl)$2 \mathrm{H}$-tetrazolium (MTS) reagents (Toronto Research Chemicals) were dissolved in DMSO at $200 \mathrm{~mm}$ and stored at $-80^{\circ} \mathrm{C}$ until use. The abbreviations used for MTS cross-linkers are described previously (Sato et al., 2006, 2008). cDNAs encoding $\mathrm{APP}_{\mathrm{NL}}$, Notch $\Delta \mathrm{E}, \mathrm{PS} 1$, and Cysless PS1 [PS1/ Cys $(-)$ ] were described previously (Kopan et al., 1996; Tomita et al., 1997; Sato et al., 2006). Single- or double-Cys mutant (mt) PS1 cDNAs were generated using a long PCR-based protocol. Maintenance of embryonic fibroblasts derived from Psen1/Psen 2 double knock-out (DKO) cells (Herreman et al., 2000), a retroviral infection system (Kitamura et al., 2003), and generation of stable infectants was performed as described previously (Watanabe et al., 2005, 2010; Sato et al., 2006, 2008). Microsome preparation and immunoblot analysis were performed as described previously (Tomita et al., 1997, 1999, 2001; Takahashi et al., 2003; Hayashi et al., 2004). For the measurement of secreted $\mathrm{A} \beta$, recombinant retrovirus encoding each Cys mt PS1 was transiently infected into DKO cells that stably expressing $\mathrm{APP}_{\mathrm{NL}}$ (Watanabe et al., 2005). After $24 \mathrm{~h}$ incubation, the conditioned medium was collected and subjected to two-site ELISAs (i.e., BAN50/BA27 and BAN50/ $\mathrm{BC} 05$ for $\mathrm{A} \beta 40$ and $\mathrm{A} \beta 42$, respectively) (Iwatsubo et al., 1994, Tomita et al., 1997, 1998, 2001). Biotinylation and competition experiments using $N$ biotinylaminoethyl methanethiosulfonate (MTSEA-biotin) in intact cells or microsome fraction have been described in detail before (Sato et al., 2006, 2008; Isoo et al., 2007). Cross-linking experiments were performed as previously described except that the microsomes were prepared from $10 \mathrm{~cm}$ dishes per single analysis. For a competition assay, sodium 2-sulfonatoethyl methanethiosulfonate (MTSES), 2-(trimethylammonium)ethyl methanethiosulfonate bromide (MSTET), or 2-(triethylammonium)ethyl methanethiosulfonate (MTS-TEAE) was preincubated with intact cells or microsomes at $2 \mathrm{~mm}$ for $5 \mathrm{~min}$ at $4^{\circ} \mathrm{C}$ and washed once before biotinylation. GSIs were preincubated with microsome aliquots for $30 \mathrm{~min}$ at room temperature before incubation with cross-linkers. The inhibitors were used at concentrations (L-685,458, $1 \mu \mathrm{M}$; DAPT, $10 \mu \mathrm{M}$; pep15, 1 $\mu \mathrm{M})$ that completely abolish the proteolytic activity of $\gamma$-secretase (Morohashi et al., 2006; Sato et al., 2006).

\section{Results}

SCAM analysis of the hydrophobic region 1 of PS1

Previous computational predictions and cell-based topological studies indicated that the hydrophobic region (HR) 1 of PS1 spans the membrane as TMD1 (Doan et al., 1996; Li and Green-

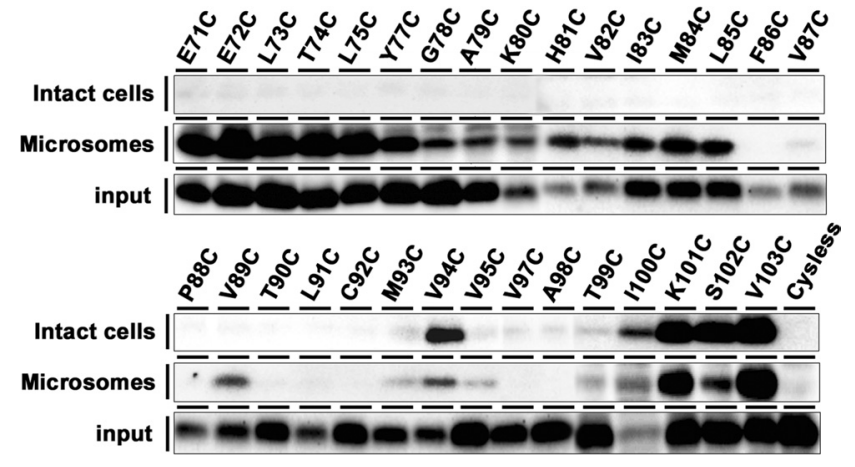

Figure 2. SCAM analysis of single-Cys mt PS1 around HR1. Intact cells (top) expressing single-Cys mt PS1 or the microsomes (middle) were incubated with MTSEA-biotin. Biotinylated proteins were precipitated with streptavidin beads and then subjected to immunoblot analysis. Cysless serves as the negative control. Amount of PS1 NTF in the input fraction is shown in the bottom panel.

wald, 1996; Lehmann et al., 1997; Nakai et al., 1999). To examine whether this region penetrates the membrane as a TMD and faces to the aqueous environment in the lipid bilayer, we constructed mutant PS1 having a single cysteine residue based on PS1/Cys(-) (single-Cys mt PS1) at 32 different amino acid residues in and around the HR1 (E71-V103), and expressed them in DKO cells (Fig. 1). We excluded V96C from further analyses, because Cys substitution of V96 resulted in loss of expression of PS1 polypeptide and failure in the restoration of the $\gamma$-secretase activity (supplemental Fig. 1, available at www.jneurosci.org as supplemental material). All the other single-Cys mt PS1 retained the protein expression, endoproteolysis, and the $\gamma$-secretase activity. SCAM analysis of the single-Cys mt PS1 in intact cells revealed that V94C, I100C, K101C, S102C, and V103C were biotinylated by MTSEA-biotin (Fig. 2, top), suggesting that these labeled residues are located in a hydrophilic milieu accessible from the extracel- 
A $\underset{\substack{0 \\ 0}}{\substack{0=j \\ 0}} \sim \mathrm{SsO}_{2} \mathrm{CH}_{3}$

MTSES

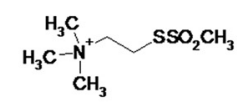

MTSET<smiles>CC[N+](CC)(CC)CCS(C)(C)C</smiles>

MTS-TEAE
B

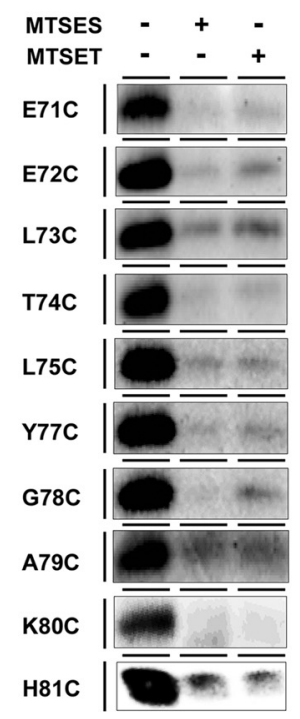

C

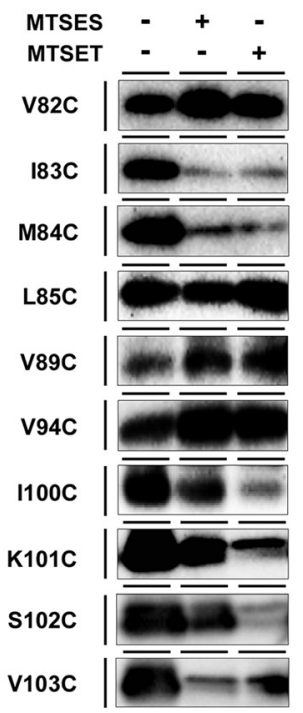

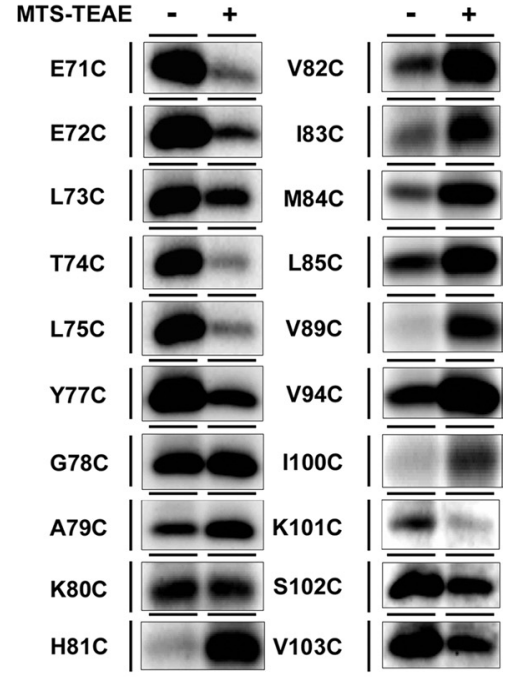

E
D

\begin{tabular}{|c|c|c|c|c|}
\hline Indicated as & MTSEA-biotin & MTSES/MTSET & MTS-TEAE & Hydrophilicity \\
\hline R & 0 & 0 & 0 & Open hydrophilic \\
\hline ৫ & 0 & 0 & $\times$ & Relatively open hydrophilic \\
\hline ○ & 0 & $\times$ & $\times$ & Closed hydrophilic \\
\hline ○ & $\times$ & $\times$ & $\times$ & Hydrophobic \\
\hline
\end{tabular}

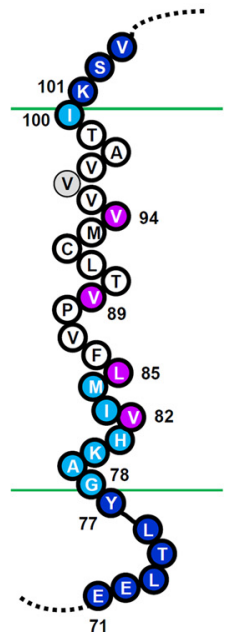

Figure 3. SCAM analysis of single-Cys mt PS1 using charged MTS reagents. $A$, The structures of the charged MTS reagents. The abbreviations are indicated below in $\boldsymbol{B}$. $\boldsymbol{B}$, Labeling competition of single-Cys mt PS1 around HR1 was examined after preincubation with negatively charged MTSES or positively charged MTSET. C, Labeling competition experiments using positively charged MTS-TEAE with a bulkier structure. $\boldsymbol{D}$, Summary of the competition experiments using charged MTS reagents. $\boldsymbol{E}$, Schematic depiction of TMD1. All charged reagents were accessible to the Cys-substituted residues indicated by blue circles. Residues whose labeling was competed by MTSES or MTSET, but not by MTS-TEAE, are indicated by sky-blue circles. Residues whose labeling was not decreased by any charged reagents are indicated by purple circles. Residues that were not labeled by MTSEA-biotin or unanalyzed are shown by black letters in white and gray circles, respectively. The predicted lipid-water interface is indicated by green lines.

Table 1. The summary of the cross-linking experiments in this study

\begin{tabular}{llll}
\hline & MTS cross-linkers & & \\
\cline { 2 - 4 } & M84C & V89C & V94C \\
\hline L383C at GXGD motif & M2M $(<5.2 \AA)$ & M2M $(<5.2 \AA)$ & M8M $(<13.0 \AA)$ \\
I387C & $M 8 M(<13.0 \AA)$ & M8M $(<13.0 \AA)$ & Not cross-linked \\
L435C at PAL motif & M11M $(<16.9 \AA)$ & M2M $(<5.2 \AA)$ & ND
\end{tabular}

"MTS cross-linkers" indicates the abbreviated name of the shortest cross-linker that enabled the production of an NTF-CTF heterodimer (i.e., M2M). Values in parentheses indicate the length of the spacer arm of the MTS cross-linkers. ND, Not determined. lular side. We next examined the accessibility of MTSEA-biotin to these residues from either the extracellular/luminal or cytosolic sides using microsome fractions from DKO cells expressing single-Cys $\mathrm{mt}$ PS1, which have been shown to be composed of a mixture of inside-out and outside-out vesicles (Sato et al., 2006). In addition to the residues that were biotinylated in intact cells, consecutive amino acid residues from E71C to L85C, as well as V89C, were positively labeled with MTSEA-biotin (Fig. 2, middle). The densitometric quantitation of each band is shown in supplemental Figure 2 (available at www.jneurosci.org as supplemental material). These results are consistent with the view that HR1 region spans the lipid bilayer with a type II orientation. Next, we analyzed the steric and electrostatic environment around the water-accessible residues using other membrane-impermeable MTS derivatives (i.e., the negatively charged MTSES and positively charged MTSET) as competitors for MTSEA-biotin (Fig. 3A, left and middle) (Sato et al., 2006, 2008). Preincubation with MTSES or MTSET failed to decrease the labeling of V82C, L85C, V89C, and V94C by MTSEA-biotin, whereas the biotinylation of other mutants was abolished by both of the charged MTS reagents (Fig. 3B). These data suggest that V82, L85, V89, and V94 are located in a closed hydrophilic environment with limited access to outside within the lipid bilayer. In contrast, other residues in which MTSES and MTSET competed for biotinylation were predicted to be facing an open hydrophilic environment. To further analyze the open hydrophilic environment in detail, we used MTS-TEAE, a sterically bulkier derivative of MTSET (Fig. $3 A$, right). As expected, preincubation with MTS-TEAE did not compete for the biotinylation of the Cys residues to which MTSES or MTSET was inaccessible (i.e., V82C, L85C, V89C, or V94C). However, MTS-TEAE failed to decrease the labeling of G78C, A79C, K80C, H81C, I83C, M84C, and I100C, although the biotinylation of these mutants was competed for that by MTSES or MTSET, suggesting that these residues are located within a relatively open, but sterically hindered, hydrophilic environment. In contrast, the labeling of other mutants (i.e., from E71C through Y77C and K101 through V103C) was decreased by preincubation with MTS-TEAE (Fig. 3C). These data indicate that the residues from E71 to Y77 and K101 to V103 are exposed to an open hydrophilic environment with unlimited access at the cytosolic and extracellular sides, respectively (for a summary, see Fig. $3 D, E)$. Strong labeling of these residues by MTSEA-biotin also supports this notion (Fig. 2; supplemental Fig. 2, available at www.jneurosci.org as supplemental material). Intriguingly, the 
biotinylation of some residues was dramatically augmented by preincubation with the membrane-impermeable MTS derivatives, presumably due to the increased availability of MTSEA-biotin by masking of the cysteines of other proteins. Together, these results suggest that the subregion of PS1 from G78 through I100 penetrates the membrane as TMD1, and that the N-terminal region of TMD1 sits in a hydrophilic, but sterically hindered, environment.

\section{Cross-linking experiments using MTS cross-linkers}

We have previously shown that the amino acid residues in TMD6 are located in proximity to those in TMD7 and TMD9, which are collectively involved in the formation of the catalytic pore, by crosslinking experiments (Sato et al., 2006, 2008). To investigate whether TMD1 also is facing to the catalytic pore, we performed cross-linking experiments using MTS cross-linkers. M2M, M3M, M4M, M6M, M8M, M11M, M14M, and M17M are sulfhydryl-to-sulfhydryl cross-linking reagents with spacer arms 5.2, 6.5, 7.8, $10.4,13.0,16.9,20.8$, and $24.7 \AA$ long, respectively (Loo and Clarke, 2001). No cross-linked products were observed by coincubation with any MTS cross-linker in samples from cells expressing singleCys mt PS1 (supplemental Fig. 3, available at www.jneurosci.org as supplemental material), in accordance with previous findings (Sato et al., 2006, 2008). We next generated double-Cys mutant PS1 bearing a pair of Cys, one located within TMD1 (M84C, V89C, or V94C) and the other in CTF, in positions previously shown to be located within the catalytic pore (i.e., L383C in TMD7, I387C, and L435C in PAL motif at TMD9) (for a summary, see Table 1). All double-Cys mt PS1 showed the $\gamma$-secretase activity (supplemental Fig. 4, available at www.jneurosci.org as supplemental material). Coincubation of all double-Cys mt PS1 (except V94C/I387C) with MTS cross-linkers yielded a novel band migrating closely to the PS1 holoprotein (Fig. 4; supplemental Fig. 5, available at www.jneurosci.org as supplemental material). Anti-PS1 NTF and CTF antibodies gave the same results, suggesting that these bands correspond to cross-linked products of NTF and CTF of PS1, in accordance with the previous observations (Sato et al., 2006, 2008). These results indicate that TMD1 faces an identical hydrophilic catalytic pore that is formed by TMD7 and TMD9. Notably, in M84C/L383C, V89C/L383C, and $\mathrm{V} 89 \mathrm{C} / \mathrm{L} 435 \mathrm{C}$, cross-linked products were observed by coincubation with M2M harboring a spacer arm $5.2 \AA$ in length. In contrast, other double-Cys mt PS1 required longer cross-linkers (i.e., M8M with spacer arm of $13.0 \AA$ in M84C/I387C, V89C/ I387C, and V94C/L383C; M11M with spacer arm of $16.9 \AA$ in M84C/L435C). Thus, M84 and V89 are predicted to be located in close proximity to the catalytic center, as L383 is located in
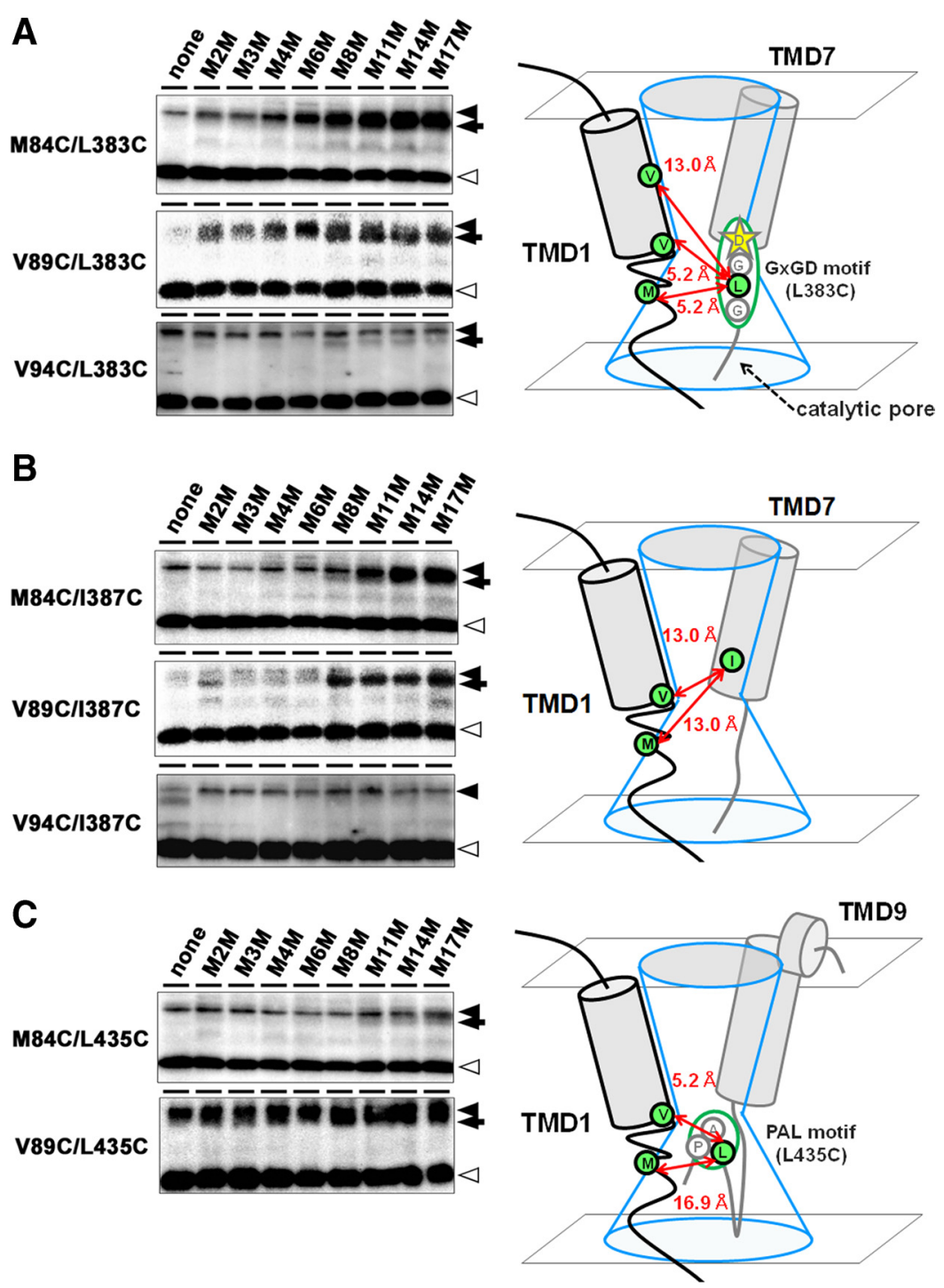

Figure 4. Cross-linking experiments of TMD1 and C-terminal residues using MTS cross-linkers. A-C, Microsome fractions from DKO cells expressing the double-Cys mt carrying one Cys residue at TMD1 and the other at L383C $(\boldsymbol{A}), \mathrm{I387C}(\boldsymbol{B})$, or L435C ( $\boldsymbol{C}$ ) were performed using anti-PS1 ${ }_{\mathrm{NT}}$ antibody. Locations of Cys substitution are shown at the left side of the panel. PS1 FL, NTF, and cross-linked product (NTF-CTF heterodimer) are shown by black arrowheads, white arrowheads, and black arrows, respectively. The predicted maximum lengths between the two residues are indicated in the schematic illustrations at the right side.

only two amino acids away from D385, the latter being one of the catalytic aspartates of PS1. These results suggest that the $\mathrm{N}$-terminal region of TMD1 participates in the formation of the hydrophilic structure that resides within the cytosolic side of the catalytic pore.

Effects of $\gamma$-secretase inhibitors on the MTS labeling of TMD1 and $\mathrm{A} \boldsymbol{\beta}$ secretion

Next, we examined the effects of three GSIs (i.e., L-685,458, pep15, and DAPT) that have been well characterized functionally and belong to distinct classes of inhibitors on the accessibility of MTSEA-biotin to substituted Cys residues as previously described (Sato et al., 2006, 2008). L-685,458 is a transition-state analog-type GSI that targets the catalytic aspartates, and directly binds NTF and CTF of PS1 (Li et al., 2000; Shearman et al., 2000). Pep15 is a helical peptide-based GSI mimicking the TMD structure, which also binds to PS1 NTF and CTF but does not share the binding site with the transition-state analog inhibitors (Das et al., 


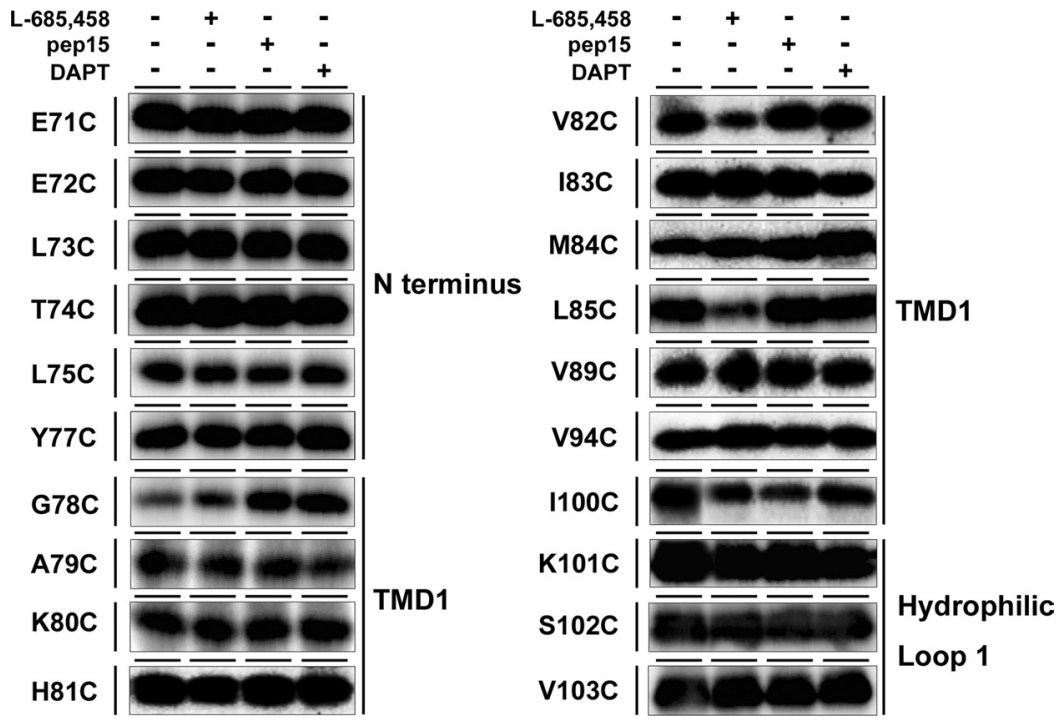

Figure 5. Labeling competition by $\gamma$-secretase inhibitors. Biotin labeling of single-Cys mt PS1 was performed after preincubation with L-685,458, pep15, or DAPT. Locations and predicted topology of Cys mutations are shown at left and right, respectively.

A

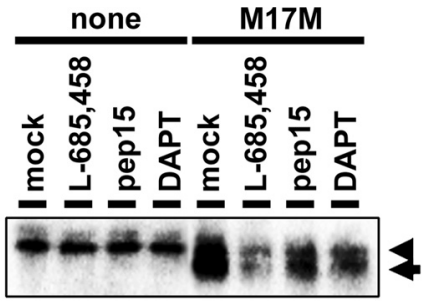

B

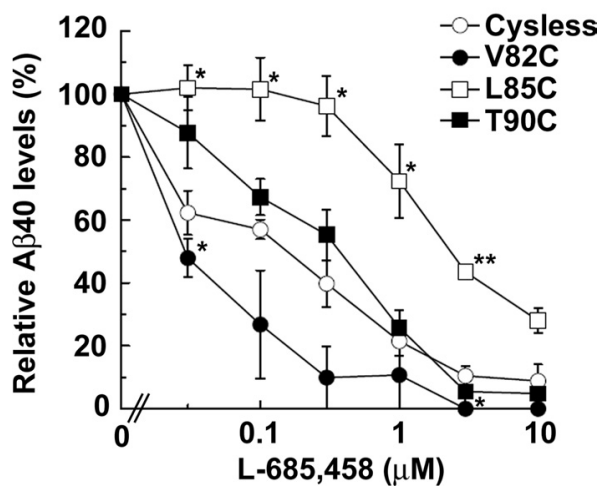

Figure 6. Effect of $\gamma$-secretase inhibitors on cross-linking and $\gamma$-secretase activity in Cys $\mathrm{mt}$ PS1. A, Cross-linking experiment on PS1 harboring double Cys (M84C and I387C) was performed in the presence of L-685,458, pep 15 or DAPT. Immunoblot analysis was performed using antiPS1 $1_{\mathrm{NT}}$ antibody. PS1 FL and cross-linked product (NTF-CTF heterodimer) are shown by black arrowheads and black arrows, respectively. $\boldsymbol{B}$, Inhibitory effect of $L-685,458$ on the A $\beta 40$ secretion from DKO cells expressing PS1/Cys(-), V82C, L85C, or T90C. Secreted A 340 in conditioned media was quantitated by ELISA $\left[n=3\right.$; mean $\pm \mathrm{SE} ;{ }^{*} p<0.05,{ }^{* *} p<0.01$ compared with PS1/Cys (-)].

2003; Kornilova et al., 2005). A dipeptidic inhibitor, DAPT binds to PS1 CTF at a site distinct from, but overlapping with, those of transition-state analogs and helical peptides (Kornilova et al., 2003, 2005; Morohashi et al., 2006). Surprisingly, all three GSIs augmented the labeling of G78C, suggesting that the water accessibility of G78 is increased in an enzymatically inactive $\gamma$-secretase. In sharp contrast, the labeling of I100C was decreased by preincubation with one or the other of GSIs. The reciprocal changes in the water accessibility of G78 and I100, which are presumed to be located at the cytoplasmic and luminal borders of TMD1, respectively, upon incubation with the GSIs, may implicate a vertical movement of TMD1 depending on the enzymatically active or inactive states of PS1. In addition, the accessibility of MTSEA-biotin to V82C and L85C was substantially decreased specifically with L-685,458 (Fig. 5), similarly to A246C, L381C, and Cys mutants around the PAL motif (Sato et al., 2006, 2008). Together with the data from cross-linking experiments, these results suggest that L-685,458 occupies the hydrophilic space formed by TMDs 1,7 , and 9 at the cytosolic side of the pore, and that V82 and L85 are involved in the binding of L-685,458. To further test this possibility, we examined the effects of GSIs on the crosslinking of $\mathrm{M} 84 \mathrm{C} / \mathrm{I} 387 \mathrm{C}$. The biotinylation of I387C single Cys mutant PS1 was not affected by preincubation of GSIs, as in M84C single Cys mutant (Fig. 5) (Sato et al., 2006). However, the amount of cross-linked products in M84C/I387C was significantly decreased by preincubation with L-685,458 (Fig. $6 A$ ). We have shown that L-685,458, which targets the catalytic site as well as the subsites of $\gamma$-secretase (Li et al., 2000), specifically affected the labeling of residues near these cross-linked residues [i.e., V82 and L85 (Fig. 5); L381 and L383 (Sato et al., 2006)]. Thus, the binding of L-685,458 might sterically interrupt the accessibility of MTS cross-linker to PS1 by occupation of the hydrophilic pore, of which the cytosolic side of TMD1 is involved in its formation. Interestingly, preincubation by DAPT or pep 15 also substantially decreased the levels of cross-linked products. The binding sites of these GSIs are overlapped, but different, from those of the transition-state analog (Das et al., 2003; Kornilova et al., 2003, 2005; Morohashi et al., 2006), and the biotinylation of the residues in TMD1 was not affected by either DAPT or pep15. Collectively, these data support the notion that DAPT and pep 15 harbor the allosteric effects on the TMD1. Finally, we examined the inhibitory potency of L-685,458 against Cys mutant of TMD1. The inhibitory potency of L-685,458 in DKO cells expressing V82C was significantly augmented, whereas that in L85C was decreased, compared with that in $\operatorname{PS} 1 / \operatorname{Cys}(-)$. In contrast, T90C, which was not labeled by MTSEA-biotin, showed a similar response to L-685,458 compared with PS1/Cys(-) (Fig. 6B), suggesting that the amino acid substitution at V82 or L85 significantly altered the structure of the binding site for L-685,458. Collectively, these observations suggest that V82 and L85 at the N-terminal region of TMD1 comprise a part of the subsite for $\gamma$-secretase within the catalytic pore.

\section{Discussion}

The structure of TMD1 of PS1 in an enzymatically active $\gamma$-secretase complex

Here, we show for the first time that TMD1 of PS1, composed of residues G78 to I100, is located in proximity to TMD7 and TMD9, and faces the catalytic pore of $\gamma$-secretase within the lipid bilayer (Fig. 7A). Amino acid alignment of PS orthologs reveals that most of the PS family members harbor a highly conserved proline-valine (PV) residues (at 88th and 89th positions in human PS1) at the center of the putative TMD1 (supplemental Fig. 
A

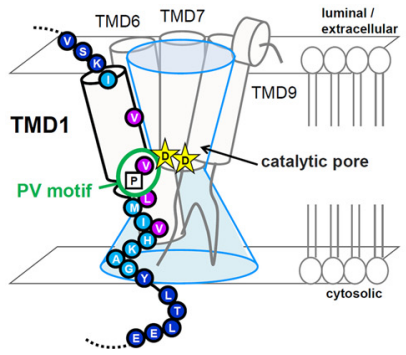

B

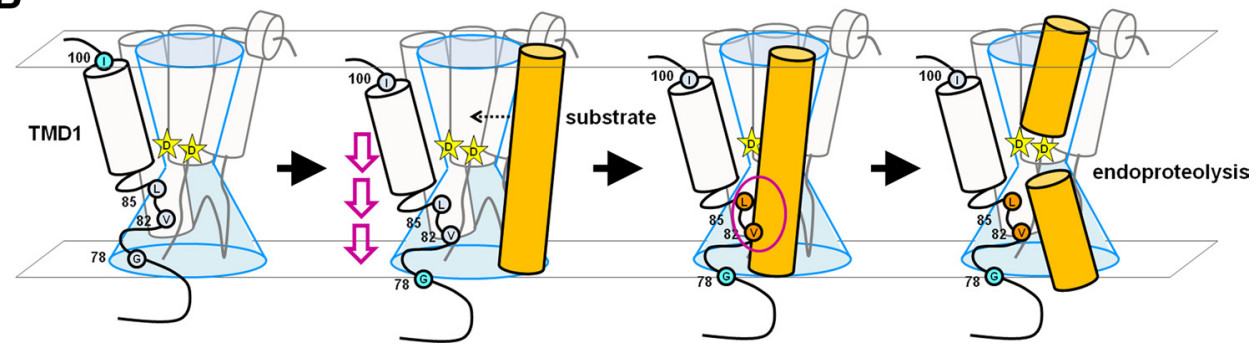

Figure 7. Structure and function of TMD1 of PS1 in relation to the $\gamma$-cleavage. $A$, Summary of SCAM analysis and a schematic depiction of the configuration of the TMD1 in relevance to the catalytic pore. Proline 88 is indicated by a square. Cys mutants that were labeled by MTSEA-biotin are shown in circle frames. Residues whose labeling was competed for by MTS-TEAE, MTSES, and MTSET are shown by blue circles. Residues whose labeling was competed for by MTSES or MTSET, but not by MTS-TEAE, are indicated by sky-blue circles. Residues whose labeling was not affected by any charged residues are indicated by purple circles. $\boldsymbol{B}$, Schematic presentation of the functional role of TMD1 in the $\gamma$-cleavage process. Binding of the substrate to the initial substrate binding site causes the piston movement (purple arrows) and changes the microenvironment of G78 and I100 at the membrane border (indicated by gray and blue circles as decreased and increased hydrophilicity, respectively). A substrate is recognized by the subsite in the catalytic pore (purple oval) where V82 and L85 at N-terminal region of TMD1 (orange circles) are involved in its formation.

6, available at www.jneurosci.org as supplemental material). We showed that V89C was accessible to MTSEA-biotin from the cytosolic side, and cross-linkable to residues of the catalytic GxGD and PAL motifs, suggesting that the PV motif in TMD1 is located in proximity to the catalytic center. Transmembrane helix kinks commonly occur at proline residues (von Heijne, 1991). It has also been suggested that mutations of the $\mathrm{N}$ - and C-terminal regions of TMD1 differentially affected the PS endoproteolysis and the $\gamma$-secretase activity (Brunkan et al., 2005). Consistently, we showed that $\mathrm{N}$ - and C-terminal portions of TMD1 divided by the PV motif exhibit distinct labeling patters by MTS reagent: consecutive residues from G78 to L85 were accessible to MTSEAbiotin, whereas V94C was the only labeled residue in the C-terminal region of TMD1. We speculate that the conserved proline 88 produces a kink in a way to separate TMD1 into two functional regions.

We thoroughly analyzed the hydrophilic environment around TMD1 and found that the cytosolic N-terminal region of the TMD1 may constitute the catalytic structure. Consecutive residues from E71C to Y77C are reactive in microsomes with all charged MTS reagents, including charged bulky MTS-TEAE, indicating that the most $\mathrm{N}$-terminal region of PS1 in a functional state is oriented to the cytosol as previously speculated (Doan et al., 1996). However, the labeling of consecutive residues from G78C to H81C, I83C, and M84C was competed with MTSES or MTSET, but not with MTS-TEAE, suggesting that they lie within a narrower hydrophilic environment. The predicted sizes of the head group of MTSET and MTS-TEAE are 5.8 and $8.0 \AA$, respectively, possibly reflecting the size of the hydrophilic environment around each modified residue. However, the successive pattern of labeling suggests that this region is not embedded in the lipid bilayer in an $\alpha$-helical structure. This is highly reminiscent of the pattern of biotinylation of the cytosolic side of TMD7, which we interpreted as a "catalytic plug" structure hung in a hydrophilic pore (Sato et al., 2006). Different competition profile (i.e., competed with MTSES and MTSET in TMD1, but not in TMD7) would reflect the heterogeneity of the hydrophilic milieu within the pore. We speculate that the residues in TMD1 with which MTS-TEAE are unreactive (i.e., G78 to L85) form a hydrophilic structure at the membrane interface of the pore. In addition, M84 was cross-linked to L383 at GxGD motif and L435 at PAL motif. Moreover, the labeling of V82C and L85C was, as in Cys mutants of the GxGD motif, specifically decreased by preincubation with L-685,458 (Fig. 5), and these mutants exhibited an altered sensitivity to L-685,458 (Fig. $6 \mathrm{~B}$ ). These data suggest that these resi- dues play a critical role in the $\gamma$-secretase activity as a subsite that is involved in substrate recognition during endoproteolysis, although an allosteric effect of L-685,458 on the biotinylation cannot be fully excluded. Collectively, these data support the notion that the cytosolic regions of TMDs 1, 7, and 9 are located in close proximity to each other and may cooperatively function as the catalytic plug.

In contrast to the N-terminal half, most residues in the C-terminal half of TMD1 were hydrophobic: only V94 was water accessible with restrictions and cross-linked with L383. However, no GSIs affected the water accessibility of V94C, suggesting that V94 directly faces the catalytic pore as a part of the pore wall, but do not participate in the formation of a subsite. Notably, the Cys substitution of V96, located at the opposite side of V94 in an $\alpha$-helical model, caused a loss of protein expression. Consistently, TMD1 is involved in the stabilization of the $\gamma$-secretase after the complex assembly is completed (Brunkan et al., 2005; Watanabe et al., 2010). Moreover, amino acid alignment also revealed that V96 is highly conserved from plants to mammals (supplemental Fig. 6, available at www.jneurosci.org as supplemental material). Thus, we speculate that the $\mathrm{C}$-terminal region of TMD1 is an essential domain for maintaining the integrity of PS1 structure via hydrophobic interactions with other TMDs. The observation that C92 in TMD1 was cross-linked with C410 or C419 in TMD8, the latter being totally embedded within the lipid bilayer (Sato et al., 2008), by oxidation-mediated crosslinking (Kornilova et al., 2006), supports this view.

We further characterized the unique feature of the catalytic pore in $\gamma$-secretase that has not been observed in other I-CLiPs. We previously reported that the extracellularly accessible L250 in TMD6 was cross-linkable with L435 at the PAL motif that is accessible only from the cytosolic side (Sato et al., 2008). In this study, again, MTS cross-linkers were able to connect the residues accessible only from the cytosol with those from the extracellular/ luminal side (i.e., M84C/I387C, V89C/I387C, and V94C/L383C) (Fig. 4). These data indicate that the catalytic pore has a connected structure through the cytosolic and extracellular/luminal sides, as suggested by the single particle analysis of the purified enzyme (Lazarov et al., 2006; Ogura et al., 2006; Osenkowski et al., 2009). This is a unique feature specific to $\gamma$-secretase/PS, since the resolved structures of other I-CLiPs (i.e., rhomboid and S2P) exhibit water-including crevasses facing only the extracellular or the cytosolic sides, respectively (Urban and Shi, 2008). The $\gamma$-secretase cleaves its substrates at multiple sites with a low sequence specificity (Qi-Takahara et al., 2005; Hemming et al., 
2008), whereas other I-CLiPs generally attack a single site in a sequence-specific manner (Ye et al., 2000; Urban and Wolfe, 2005). It is interesting to speculate that the pore structure is related to the multistep, as well as multisite, $\gamma$-secretase-mediated cleavage process.

\section{Implications for the mechanism of intramembrane proteolysis}

A previous study has implicated TMD1 in the substrate binding of $\gamma$-secretase (Annaert et al., 2001). Photoaffinity labeling and cross-competition experiments have suggested that the substrate-mimic helical peptides target the initial substratebinding site in PS, which is predicted to be distinct from the catalytic site (Das et al., 2003; Kornilova et al., 2005; Imamura et al., 2009). What, then, is the role of TMD1 in the substrate binding? In this study, however, we failed to detect any specific effects of pep 15 on the labeling of TMD1 residues. Rather, competition experiments suggested that TMD1 faces the catalytic pore and functions as a subsite. Notably, Cys substitution of F86 caused an almost complete loss of $\mathrm{A} \beta$ generation as well as E-cadherin cleavage, whereas the Notch-cleaving activity was retained in this mutant (supplemental Figs. 1, 7, available at www.jneurosci.org as supplemental material), suggesting that TMD1 plays some role in substrate selection. Nonetheless, we cannot exclude the possibility that TMD1 is directly involved in the substrate binding, in which TMD-TMD interactions at a hydrophobic interface are required, since SCAM analysis using the hydrophilic MTS reagent is unable to detect the hydrophobic interactions.

We raise the possibility that TMD1 dynamically moves in a vertical direction upon substrate binding. We showed that GSIs augmented the hydrophilicity of G78 located at the cytoplasmmembrane border of TMD1. Simultaneously, all three GSIs significantly decreased the water accessibility of I100C, which is positioned at the extracellular membrane interface of TMD1. Altogether, these changes in the labeling pattern suggest that GSIs induced a vertical motion of TMD1 toward the cytosolic side in an allosteric manner. Binding of transition-state analog-type GSIs was shown to stabilize the substrate-PS interaction at the initial substrate binding site, the target of the helical peptide-type GSIs (Esler et al., 2002). These findings may suggest that the vertical motion caused by GSIs was elicited by the occupancy of the initial substrate binding site. Similar motion in TMD caused by ligand binding has been documented as "piston movement" in integrins, bacterial chemoreceptors, $\beta 2$-adrenergic receptor, and $\mathrm{Ca}^{2+}$-ATPase pump (Williams et al., 1994; Møller et al., 2005; Spijker et al., 2006; Hazelbauer and Lai, 2010). Also, changes in membrane potential trigger a similar motion of TMD in voltagedependent potassium channels (Gandhi et al., 2003). To our knowledge, this is the first biochemical indication of a motion of PS1 in a membrane-embedded, functional state (for a summary, see Fig. $7 B$ ).

In sum, we have revealed that TMD1 is a functional component of the catalytic pore structure of the $\gamma$-secretase and suggested the dynamic motion of TMD1 during the intramembranecleaving process. However, it is unclear whether this motion is a dynamic process correlated with endoproteolysis, and it remains possible that GSIs affect the water accessibility of these residues by an independent mechanism. Nonetheless, further integrated analysis (i.e., molecular dynamics simulation with lipid molecule), in addition to conventional structural analysis of PS at a higher resolution, will provide crucial information for the rational design of GSIs for AD treatment.

\section{References}

Annaert WG, Esselens C, Baert V, Boeve C, Snellings G, Cupers P, Craessaerts K, De Strooper B (2001) Interaction with telencephalin and the amyloid precursor protein predicts a ring structure for presenilins. Neuron 32:579-589.

Brunkan AL, Martinez M, Wang J, Walker ES, Beher D, Shearman MS, Goate AM (2005) Two domains within the first putative transmembrane domain of presenilin 1 differentially influence presenilinase and $\gamma$-secretase activity. J Neurochem 94:1315-1328.

Das C, Berezovska O, Diehl TS, Genet C, Buldyrev I, Tsai JY, Hyman BT, Wolfe MS (2003) Designed helical peptides inhibit an intramembrane protease. J Am Chem Soc 125:11794-11795.

De Strooper B, Vassar R, Golde T (2010) The secretases: enzymes with therapeutic potential in Alzheimer disease. Nat Rev Neurol 6:99-107.

Doan A, Thinakaran G, Borchelt DR, Slunt HH, Ratovitsky T, Podlisny M, Selkoe DJ, Seeger M, Gandy SE, Price DL, Sisodia SS (1996) Protein topology of presenilin 1. Neuron 17:1023-1030.

Dovey HF, John V, Anderson JP, Chen LZ, de Saint Andrieu P, Fang LY, Freedman SB, Folmer B, Goldbach E, Holsztynska EJ, Hu KL, JohnsonWood KL, Kennedy SL, Kholodenko D, Knops JE, Latimer LH, Lee M, Liao Z, Lieberburg IM, Motter RN, et al. (2001) Functional $\gamma$-secretase inhibitors reduce $\beta$-amyloid peptide levels in brain. J Neurochem 76:173-181.

Esler WP, Kimberly WT, Ostaszewski BL, Ye W, Diehl TS, Selkoe DJ, Wolfe MS (2002) Activity-dependent isolation of the presenilin- $\gamma$-secretase complex reveals nicastrin and a $\gamma$ substrate. Proc Natl Acad Sci U S A 99:2720-2725.

Fuwa H, Takahashi Y, Konno Y, Watanabe N, Miyashita H, Sasaki M, Natsugari H, Kan T, Fukuyama T, Tomita T, Iwatsubo T (2007) Divergent synthesis of multifunctional molecular probes to elucidate the enzyme specificity of dipeptidic $\gamma$-secretase inhibitors. ACS Chem Biol 2:408-418.

Gandhi CS, Clark E, Loots E, Pralle A, IsacoffEY (2003) The orientation and molecular movement of a $\mathrm{K}(+)$ channel voltage-sensing domain. Neuron 40:515-525.

Haass C, Selkoe DJ (2007) Soluble protein oligomers in neurodegeneration: lessons from the Alzheimer's amyloid $\beta$-peptide. Nat Rev Mol Cell Biol 8:101-112.

Hayashi I, Urano Y, Fukuda R, Isoo N, Kodama T, Hamakubo T, Tomita T, Iwatsubo $\mathrm{T}$ (2004) Selective reconstitution and recovery of functional $\gamma$-secretase complex on budded baculovirus particles. J Biol Chem 279:38040-38046.

Hazelbauer GL, Lai WC (2010) Bacterial chemoreceptors: providing enhanced features to two-component signaling. Curr Opin Microbiol 13:124-132.

Hemming ML, Elias JE, Gygi SP, Selkoe DJ (2008) Proteomic profiling of $\gamma$-secretase substrates and mapping of substrate requirements. PLoS Biol 6:e257.

Herreman A, Serneels L, Annaert W, Collen D, Schoonjans L, De Strooper B (2000) Total inactivation of $\gamma$-secretase activity in presenilin-deficient embryonic stem cells. Nat Cell Biol 2:461-462.

Imamura Y, Watanabe N, Umezawa N, Iwatsubo T, Kato N, Tomita T, Higuchi $\mathrm{T}$ (2009) Inhibition of $\gamma$-secretase activity by helical $\beta$-peptide foldamers. J Am Chem Soc 131:7353-7359.

Isoo N, Sato C, Miyashita H, Shinohara M, Takasugi N, Morohashi Y, Tsuji S, Tomita $\mathrm{T}$, Iwatsubo $\mathrm{T}$ (2007) A $\beta 42$ overproduction associated with structural changes in the catalytic pore of $\gamma$-secretase: common effects of Pen-2 N-terminal elongation and fenofibrate. J Biol Chem 282:12388-12396.

Iwatsubo T, Odaka A, Suzuki N, Mizusawa H, Nukina N, Ihara Y (1994) Visualization of $A \beta 42(43)$ and $A \beta 40$ in senile plaques with end-specific $\mathrm{A} \beta$-monoclonals: Evidence that an initially deposited species is $\mathrm{A} \beta 42(43)$. Neuron 13:45-53.

Kaback HR, Sahin-Tóth M, Weinglass AB (2001) The kamikaze approach to membrane transport. Nat Rev Mol Cell Biol 2:610-620.

Kan T, Tominari Y, Rikimaru K, Morohashi Y, Natsugari H, Tomita T, Iwatsubo T, Fukuyama T (2004) Parallel synthesis of DAPT derivatives and their $\gamma$-secretase-inhibitory activity. Bioorg Med Chem Lett 14:1983-1985.

Karlin A, Akabas MH (1998) Substituted-cysteine accessibility method. Methods Enzymol 293:123-145.

Kitamura T, Koshino Y, Shibata F, Oki T, Nakajima H, Nosaka T, Kumagai H 
(2003) Retrovirus-mediated gene transfer and expression cloning: powerful tools in functional genomics. Exp Hematol 31:1007-1014.

Kopan R, Schroeter EH, Weintraub H, Nye JS (1996) Signal transduction by activated mNotch: importance of proteolytic processing and its regulation by the extracellular domain. Proc Natl Acad Sci U S A 93:1683-1688.

Kornilova AY, Das C, Wolfe MS (2003) Differential effects of inhibitors on the $\gamma$-secretase complex. Mechanistic implications. J Biol Chem 278:16470-16473.

Kornilova AY, Bihel F, Das C, Wolfe MS (2005) The initial substratebinding site of $\gamma$-secretase is located on presenilin near the active site. Proc Natl Acad Sci U S A 102:3230-3235.

Kornilova AY, Kim J, Laudon H, Wolfe MS (2006) Deducing the transmembrane domain organization of presenilin- 1 in $\gamma$-secretase by cysteine disulfide cross-linking. Biochemistry 45:7598-7604.

Lazarov VK, Fraering PC, Ye W, Wolfe MS, Selkoe DJ, Li H (2006) Electron microscopic structure of purified, active $\gamma$-secretase reveals an aqueous intramembrane chamber and two pores. Proc Natl Acad Sci U S A 103:6889-6894.

Lehmann S, Chiesa R, Harris DA (1997) Evidence for a six-transmembrane domain structure of presenilin 1. J Biol Chem 272:12047-12051.

Li X, Greenwald I (1996) Membrane topology of the C. elegans SEL-12 presenilin. Neuron 17:1015-1021.

Li YM, Xu M, Lai MT, Huang Q, Castro JL, DiMuzio-Mower J, Harrison T, Lellis C, Nadin A, Neduvelil JG, Register RB, Sardana MK, Shearman MS, Smith AL, Shi XP, Yin KC, Shafer JA, Gardell SJ (2000) Photoactivated $\gamma$-secretase inhibitors directed to the active site covalently label presenilin 1. Nature 405:689-694.

Loo TW, Clarke DM (2001) Determining the dimensions of the drugbinding domain of human P-glycoprotein using thiol cross-linking compounds as molecular rulers. J Biol Chem 276:36877-36880.

Møller JV, Nissen P, Sørensen TL, le Maire M (2005) Transport mechanism of the sarcoplasmic reticulum $\mathrm{Ca}^{2+}$-ATPase pump. Curr Opin Struct Biol 15:387-393.

Morohashi Y, Kan T, Tominari Y, Fuwa H, Okamura Y, Watanabe N, Sato C, Natsugari H, Fukuyama T, Iwatsubo T, Tomita T (2006) C-terminal fragment (CTF) of presenilin is the molecular target of a dipeptidic $\gamma$-secretase-specific inhibitor DAPT ( $N$-[N-(3,5-difluorophenacetyl)- $L$ alanyl]-S-phenylglycine $t$-butyl ester). J Biol Chem 281:14670-14676.

Nakai T, Yamasaki A, Sakaguchi M, Kosaka K, Mihara K, Amaya Y, Miura S (1999) Membrane topology of Alzheimer's disease-related presenilin 1. Evidence for the existence of a molecular species with a seven membranespanning and one membrane-embedded structure. J Biol Chem 274:23647-23658.

Ogura T, Mio K, Hayashi I, Miyashita H, Fukuda R, Kopan R, Kodama T, Hamakubo T, Iwatsubo T, Iwastubo T, Tomita T, Sato C (2006) Threedimensional structure of the $\gamma$-secretase complex. Biochem Biophys Res Commun 343:525-534.

Osenkowski P, Li H, Ye W, Li D, Aeschbach L, Fraering PC, Wolfe MS, Selkoe DJ, Li H (2009) Cryoelectron microscopy structure of purified gammasecretase at 12 angstroms resolution. J Mol Biol 385:642-652.

Qi-Takahara Y, Morishima-Kawashima M, Tanimura Y, Dolios G, Hirotani N, Horikoshi Y, Kametani F, Maeda M, Saido TC, Wang R, Ihara Y (2005) Longer forms of amyloid $\beta$ protein: implications for the mechanism of intramembrane cleavage by $\gamma$-secretase. J Neurosci 25:436-445.

Sato C, Morohashi Y, Tomita T, Iwatsubo T (2006) Structure of the catalytic pore of $\gamma$-secretase probed by the accessibility of substituted cysteines. J Neurosci 26:12081-12088.

Sato C, Takagi S, Tomita T, Iwatsubo T (2008) The C-terminal PAL motif and transmembrane domain 9 of presenilin 1 are involved in the formation of the catalytic pore of the $\gamma$-secretase. J Neurosci 28:6264-6271.

Shearman MS, Beher D, Clarke EE, Lewis HD, Harrison T, Hunt P, Nadin A, Smith AL, Stevenson G, Castro JL (2000) L-685,458, an aspartyl protease transition state mimic, is a potent inhibitor of amyloid $\beta$-protein precursor $\gamma$-secretase activity. Biochemistry 39:8698-8704.

Sobhanifar S, Schneider B, Löhr F, Gottstein D, Ikeya T, Mlynarczyk K, Pulawski W, Ghoshdastider U, Kolinski M, Filipek S, Güntert P, Bernhard F,
Dötsch V (2010) Structural investigation of the C-terminal catalytic fragment of presenilin 1. Proc Natl Acad Sci U S A 107:9644-9649.

Spijker P, Vaidehi N, Freddolino PL, Hilbers PA, Goddard WA 3rd (2006) Dynamic behavior of fully solvated $\beta 2$-adrenergic receptor, embedded in the membrane with bound agonist or antagonist. Proc Natl Acad Sci U S A 103:4882-4887.

Takahashi Y, Hayashi I, Tominari Y, Rikimaru K, Morohashi Y, Kan T, Natsugari H, Fukuyama T, Tomita T, Iwatsubo T (2003) Sulindac sulfide is a noncompetitive $\gamma$-secretase inhibitor that preferentially reduces $\mathrm{A} \beta 42$ generation. J Biol Chem 278:18664-18670.

Takasugi N, Tomita T, Hayashi I, Tsuruoka M, Niimura M, Takahashi Y, Thinakaran G, Iwatsubo T (2003) The role of presenilin cofactors in the $\gamma$-secretase complex. Nature 422:438-441.

Thinakaran G, Regard JB, Bouton CM, Harris CL, Price DL, Borchelt DR, Sisodia SS (1998) Stable association of presenilin derivatives and absence of presenilin interactions with APP. Neurobiol Dis 4:438-453.

Tolia A, Chávez-Gutiérrez L, De Strooper B (2006) Contribution of presenilin transmembrane domains 6 and 7 to a water-containing cavity in the $\gamma$-secretase complex. J Biol Chem 281:27633-27642.

Tolia A, Horré K, De Strooper B (2008) Transmembrane domain 9 of presenilin determines the dynamic conformation of the catalytic site of $\gamma$-secretase. J Biol Chem 283:19793-19803.

Tomita T (2009) Secretase inhibitors and modulators for Alzheimer's disease treatment. Expert Rev Neurother 9:661-679.

Tomita T, Maruyama K, Saido TC, Kume H, Shinozaki K, Tokuhiro S, Capell A, Walter J, Grünberg J, Haass C, Iwatsubo T, Obata K (1997) The presenilin 2 mutation (N141I) linked to familial Alzheimer disease (Volga German families) increases the secretion of amyloid $\beta$ protein ending at the 42nd (or 43rd) residue. Proc Natl Acad Sci U S A 94:2025-2030.

Tomita T, Tokuhiro S, Hashimoto T, Aiba K, Saido TC, Maruyama K, Iwatsubo $\mathrm{T}$ (1998) Molecular dissection of domains in mutant presenilin 2 that mediate overproduction of amyloidogenic forms of amyloid $\beta$ peptides. Inability of truncated forms of PS2 with familial Alzheimer's disease mutation to increase secretion of A $\beta 42$. J Biol Chem 273:21153-21160.

Tomita T, Takikawa R, Koyama A, Morohashi Y, Takasugi N, Saido TC, Maruyama K, Iwatsubo T (1999) C terminus of presenilin is required for overproduction of amyloidogenic $\mathrm{A} \beta 42$ through stabilization and endoproteolysis of presenilin. J Neurosci 19:10627-10634.

Tomita T, Watabiki T, Takikawa R, Morohashi Y, Takasugi N, Kopan R, De Strooper B, Iwatsubo T (2001) The first proline of PALP motif at the C terminus of presenilins is obligatory for stabilization, complex formation, and $\gamma$-secretase activities of presenilins. J Biol Chem 276:33273-33281.

Urban S, Shi Y (2008) Core principles of intramembrane proteolysis: comparison of rhomboid and site-2 family proteases. Curr Opin Struct Biol $18: 432-441$.

Urban S, Wolfe MS (2005) Reconstitution of intramembrane proteolysis in vitro reveals that pure rhomboid is sufficient for catalysis and specificity. Proc Natl Acad Sci U S A 102:1883-1888.

von Heijne G (1991) Proline kinks in transmembrane $\alpha$-helices. J Mol Biol 218:499-503.

Watanabe N, Tomita T, Sato C, Kitamura T, Morohashi Y, Iwatsubo T (2005) Pen-2 is incorporated into the $\gamma$-secretase complex through binding to transmembrane domain 4 of presenilin 1. J Biol Chem 280:41967-41975.

Watanabe N, Takagi S, Tominaga A, Tomita T, Iwatsubo T (2010) Functional analysis of the transmembrane domains of presenilin 1: Participation of transmembrane domains 2 and 6 in the formation of initial substrate-binding site of $\gamma$-secretase. J Biol Chem 285:19738-19746

Williams MJ, Hughes PE, O’Toole TE, Ginsberg MH (1994) The inner world of cell adhesion: integrin cytoplasmic domains. Trends Cell Biol 4:109-112.

Wolfe MS, Kopan R (2004) Intramembrane proteolysis: theme and variations. Science 305:1119-1123.

Ye J, Davé UP, Grishin NV, Goldstein JL, Brown MS (2000) Asparagineproline sequence within membrane-spanning segment of SREBP triggers intramembrane cleavage by site- 2 protease. Proc Natl Acad Sci U S A 97:5123-5128. 